\title{
SELF-GUIDING OF HIGH-INTENSITY LASER PULSES FOR LASER WAKE FIELD ACCELERATION
}

\author{
D. Umstader and X. Liu \\ Center for Ultrafast Optical Science \\ University of Michigan, Ann Arbor, MI 48109-2099
}

\begin{abstract}
A means of self-guiding an ultrashort and high-intensity laser pulse is demonstrated both experimentally and numerically. Its relevance to the laser wake field accelerator concept is discussed. Self-focusing and multiple foci formation are observed when a high peak power $(P>100$ GW), $1 \mu \mathrm{m}$, subpicosecond laser is focused onto various gases (air or hydrogen). It appears to result from the combined effects of self-focusing by the gas, and de-focusing both by diffraction and the plasma formed in the central high-intensity region. Quasi-stationary computer simulations show the same multiple foci behavior as the experiments. The results suggest much larger nonlinear electronic susceptibilities of a gas near or undergoing ionization in the high field of the laser pulse. Although self-guiding of a laser beam by this mechanism appears to significantly extend its high-intensity focal region, small-scale self-focusing due to beam non-uniformity is currently a limitation.
\end{abstract}




\section{INTRODUCTION}

With the recent development of compact ultra-short pulse and high intensity lasers[1], proof-of-principle tests of proposed novel laser-plasma electron accelerators[2][4] are now feasible. A practical laser accelerator, however, will require a long high-intensity laser-plasma interaction region, which has yet to be produced. Thus a means of self-guiding intense lasers must be found. Towards this end, self-guiding mechanisms involving laser-plasma effects[6], such as relativistic self-focusing and cavitation, have been investigated[3]. Recently, however, it has been shown theoretically[4] that the conditions required for self-focusing a pulse with a Gaussian temporal profile by these methods are incompatible with those required for laser wake field acceleration.

Laser wake field acceleration requires high-intensity ultra-short laser pulses with laser pulse widths $\left(\tau_{l}\right)$ satisfying the condition $\tau_{l} \sim \tau_{p}$, where $\tau_{p}=2 \pi / \omega_{p}$ is the plasma period, the inverse of the plasma frequency $\left(\omega_{p}=4 \pi n_{f}^{2} / m\right)$. This places a restriction on the density of free electrons $\left(n_{f}\right)$ for a given laser pulse width. For instance, for a picosecond laser, the suitable plasma density is $n_{f}=10^{16} \mathrm{~cm}^{-3}$. Because of collective plasma effects, however, in order for the above methods of self-guiding to work, several plasma periods are required in a pulse width. In other words relativistic self-focusing will only work at densities that are higher than are suitable for laser wake field acceleration.

We report in this paper the observation, both experimentally and numerically, of self-focusing and multiple-foci formation of subpicosecond laser pulses, by a method that does not require several plasma periods in a pulse width. It involves both plasma and, in addition, neutral gas effects on the beam propagation. Although multiple sparks produced by longer laser pulses at atmospheric pressures were observed in gas breakdown experiments[7], and self-focusing was identified as the mechanism, here we concentrate on self-guiding under conditions relevant to laser wake field acceleration, i.e., with ultra-short laser pulses and lower gas densities.

Self-focusing or self-defocusing, in either atomic or ionized media, originates from a radial intensity-dependent refractive index gradient. Self-focusing occurs when a negative gradient acts as a positive lens, and vice versa for self-defocusing. The mechanism responsible for the index change depends on the laser pulse duration and intensity. For $\mathrm{cw}$ and long pulses, thermal[8], ponderomotive[5], and molecular orientational nonlinear effects[9] are dominant. For pulses of picosecond and shorter duration, only the electronic nonlinear susceptibility and ionization are important[9]. Finally, at the highest laser intensities, relativistic effects must be considered[6].

Self-guiding by the mechanism that we will be discussing arises naturally when an intense laser is focused into a gas. A plasma will be formed wherever the intensity exceeds the ionization threshold. In the gas region, a negative radial index gradient will be created by the intensity-dependence of the nonlinear susceptiblity, focusing the beam. In the plasma region, a positive 
radial index gradient will be created by the intensity-dependence of the ionization, defocusing the beam. A focused beam will also defocus due to diffraction even without an index gradient. A combination of these three effects may result in multiple self-focusing.

\section{EXPERIMENTAL ARRANGEMENT}

A Nd:glass laser system[10], based on the principle of chirped-pulse amplification[11], was used in the experiment. Laser pulses of $1.05-\mu \mathrm{m}$ wavelength, $400-\mathrm{fs}$ duration, and $10^{5}$-intensity contrast ratio were focused into a chamber that was backfilled with either air or hydrogen. Lenses of either of two focal lengths were used, $20 \mathrm{~cm}(\mathrm{f} / 9)$, or $40 \mathrm{~cm}(\mathrm{f} / 18)$. Pressures of $1-500$ Torr in the chamber were measured to within an accuracy of $0.5 \%$ with a capacitance manometer. When focused into an evacuated chamber using the $f / 9$ lens, the laser beam was measured to have a two-and-a-half times diffraction-limited spot size of about $40 \mu \mathrm{m}$, a peak intensity up to $10^{16} \mathrm{~W} / \mathrm{cm}^{2}$, and an energy per pulse in the range of $20-140 \mathrm{~mJ}$. The laser power used in the experiment was kept below the threshold for relativistic self-focusing[6].

Without considering the plasma defocusing effect, the power level of the laser pulses in these experiments exceeds the critical power, $P_{c r}=\lambda^{2} c / 16 \pi^{2} n_{2}[12]$, for self-focusing to occur in the medium with nonlinear refractive index $n_{2}$. This self-focusing would lead to catastrophic intensities in the focused region. However, plasma defocusing prevents such a process from occuring, and actually leads to multiple foci formation, as shown in both the experiments and numerical simulations.

In order to image the location of the foci, light emitted from the laser-produced plasma was recorded with a digital CCD camera looking perpendicular to the laser axis. An infrared filter was placed in front of the CCD camera to block any stray laser light.

\section{EXPERIMENTAL RESULTS}

At low gas densities ( $p \leq 25$ Torr) and low laser energies, no evidence for self-focusing was observed. Images recorded under these conditions (using the f/9 lens) indicate the formation of a plasma column of length roughly twice the Rayleigh range, as.shown in Fig. 1(a). The midpoint of this plasma column serves as reference point indicating the location of the best focus in the case when no significant self-focusing or self-defocusing occurs. Beam propagation was investigated under various conditions, by varying either the laser intensity, polarization state, focusing geometry, or the gas density.

As the gas density was increased to about $p=50$ Torr, the plasma column moved backward from the reference point toward the incident laser beam, and a second focus developed (Fig. 1(b)). As the gas density increased even further yet, three or more foci appeared (Fig. 1(c)-(d)). The energy threshold for $p=50$ Torr appears to be about $40 \mathrm{~mJ}$, which corresponds to a peak power of about $100 \mathrm{GW}$. The number of self-focused foci and their positions varied from shot to 
shot as the energy in the laser pulse fluctuated. The $f=40 \mathrm{~cm}$ lens produced similar effects, with correspondingly longer focal region (Fig. 2(a) - (d)). The general behavior of the focus moving toward the incident laser beam with increasing density is expected because as the gas density increases, so does the electronic nonlinear refractive index, and the resulting positive lens effect causes the beam to focus earlier.

The propagation was sensitive to the laser intensity and beam quality, but not polarization. Linear polarization, both perpendicular and parallel to the CCD camera axis, as well as circular polarization, produced similar results. When the pressure was held constant at some value ( $p>200$ Torr), self-focusing was observed for laser energies varying between $30-120 \mathrm{~mJ}$, generally with higher energies producing more foci. Some nonuniformity of the laser beam profile was present, as evidenced by the on-axis image of the laser focal spot made when the laser was focused into vacuum, shown in Fig. 3(a). Because the incident laser beam has spatial nonuniformity, and as such is unstable against small scale self-focusing when propagated in a nonlinear medium, the beam will eventually break-up. An on-axis image made with the chamber filled with 50 Torr of hydrogen (Fig. 3(b)) confirmed this. The trajectory of some of the beam foci appear to be curved in Figs. 1 and 2. We believe this is caused by nonlinear bending also due to the nonuniformity of the incident laser beam[13].

\section{THEORETICAL MODEL}

We modeled the laser beam propagation taking into consideration the above-mentioned effects. A focused high-intensity laser pulse may produce a plasma near the center of the beam by a number of ionization mechanisms. For our laser parameters, tunneling ionization dominates. We use only hydrogen with a single electron in the model for simplicity. Because of the short laser pulse duration, we also neglect any thermodynamic motion of the plasma during the interaction. The medium is assumed to be a mixture of free and bound electrons. If we follow a particular point in the pulse's temporal envelope (or assume the quasi-stationary condition), the free electron density as a function of the laser field can be approximated by $n_{f}=n_{t} \exp \left[-E_{s} / E(\mathbf{r})\right]$ and the bound electron density by

$$
n_{b}=n_{t}-n_{f}=n_{t}\left(1-\exp \left[-E_{s} / E(\mathbf{r})\right]\right)
$$

where $E=E(\mathbf{r})$ is the laser electric field, $E$, the saturation field for ionization, and $n_{t}$ the total electron density, which is proportional to the original gas density (pressure).

The bound electrons, in the high laser field, contribute to the nonlinear refractive index of the medium. Usually the nonlinear index can be written as $\eta_{N L}(\mathbf{r})=n_{2}|E(\mathbf{r})|^{2}$, where $n_{2}$, the nonlinear refractive index constant, is positive and proportional to the bound electron density. The nonlinear index, $n_{2}$, is 
related to the third order nonlinear susceptibility $\left(\chi^{(3)}\right)$ by,

$$
n_{2}=\frac{12 \pi n_{b}}{\eta} \chi_{1111}^{(3)}(-\omega ; \omega, \omega,-\omega)=n_{2}^{\prime} \times n_{b}
$$

The free electrons, on the other hand, contribute to the plasma index of refraction. This is given by $\eta_{\text {plasma }}=\sqrt{1-n_{f} / n_{c}} \simeq 1-(1 / 2)\left(n_{f}(\mathbf{r}) / n_{c}\right)$ when the density of free electrons is much less than the critical density, or, $n_{f}(\mathbf{r}) \ll n_{c}$ . The combined index of refraction is then given by $\eta=1+n_{2}|E(\mathbf{r})|^{2}-(1 / 2)\left(n_{f}(\mathrm{r}) / n_{c}\right)$. In the paraxial approximation, we can thus write the nonlinear Schroedinger equation for the complex amplitude of the laser field $E=A \exp \left[i\left(k_{o} z-\omega t\right)\right]$ as

$$
i \frac{\partial A}{\partial z}=-\frac{1}{2 k_{o}} \nabla_{\perp}^{2} A-k_{o}\left(n_{2}|A|^{2}-\frac{1}{2} \frac{n_{f}}{n_{c}}\right) A .
$$

Substituting $n_{2}$ and $n_{f}$ as functions of the laser field amplitude, we get:

$$
i \frac{\partial A}{\partial z}=-\frac{1}{2 k_{o}} \nabla_{\perp}^{2} A-k_{o}\left\{n_{2}^{\prime} n_{t}\left(1-\exp \left[-\frac{E_{s}}{|A(\mathbf{r})|}\right]\right)|A|^{2}-\frac{n_{t}}{2 n_{c}} \exp \left[-\frac{E_{s}}{|A(\mathbf{r})|}\right]\right\} A \text {. }
$$

The first term on the right hand side represents beam diffraction, the second, self-focusing, and the third, plasma defocusing.

\section{SIMULATION RESULTS}

Rather than attempting an analytical solution of an equation as nonlinear as Eq. (4), we instead solved it numerically. A cylindrically symmetric radial beam profile was assumed. Some computer simulation results are presented in Fig. 4(a) - (d), in which it was further assumed that the initial beam profile was Gaussian. The ionization saturation intensity, $I_{s}=(c / 8 \pi) E_{s}^{2}$, is taken to be $I_{s}=10^{15}$ $\mathrm{W} / \mathrm{cm}^{2}[14][15] . I_{s}$ can also be estimated from the tunneling ionization rate[16]

$$
\nu=3.8 \times 10^{11} \epsilon_{I}^{1 / 2}\left(\frac{I}{\epsilon_{I}^{3}}\right)^{1 / 4} \exp \left[-2.4 \times 10^{6}\left(\frac{\epsilon_{I}^{3}}{I}\right)^{1 / 2}\right],
$$

where $\epsilon_{I}$ is the ionization energy, expressed in $\mathrm{eV}$, of the gas molecule $(\sim 14 \mathrm{eV}$ for hydrogen). For subpicosecond pulses $\nu$ must be on the order of $10^{13} \mathrm{sec}^{-1}$ for a significant portion of the gas to be ionized, which also gives $I_{s}$ to be on the order of $10^{15} \mathrm{~W} / \mathrm{cm}^{2}$. It can be seen from these figures that for certain range of values of $n_{2}=n_{2}^{\prime} n_{t}$ in Eq. (4), the simulation results show the same behavior as the experiments, i.e., self-focusing, backward movement of the focus, and multiple foci formation.

It was found from these simulations that, by using values of $n_{2}$ that were measured at intensities much lower than the ionization threshold[17], the laser never reaches its vacuum spotsize or peak intensity (Fig. 4(b)). Clamping of the 
peak intensity, and hence maximum plasma density, has been observed both experimentally and numerically in $10.6-\mu \mathrm{m}$ laser interactions[18]. However, the self-guiding effects observed in our experiments are reproduced in the simulations only when much larger values of $n_{2}$ are used, about an order of magnitude larger. This may be explained by the fact that when the gas is close to ionization, the bound electrons become highly anharmonic, and higher order nonlinear effects may become important. In this case, higher order terms in the intensity should be included in the nonlinear index, $\eta_{N L}$, as

$$
\eta_{N L}=\left(n_{2}+n_{4}|E|^{2}+\cdots\right)|E|^{2} .
$$

On the other hand, the perturbative approach to the nonlinear susceptibility in the high laser field can totally break down, as evidenced in above threshold ionization studies[19]. Therefore the above power expansion in terms of $|E|^{2}$ may not be applicable in the high field limit. The nonlinear properties of the gas may be very different compared to the lower field situations, and warrant further investigations. Also, the large nonlinear current predicted to result when a gas is undergoing ionization-specifically tunneling ionization[20]-may also be important for the laser beam propagation. Finally, to better simulate the experiment, attenuation, and both the spatial and temporal shape of the laser pulse must be considered, all of which limit the distance the beam can be guided.

\section{RELEVANCE TO LASER WAKE FIELD ACCELERATION}

In this section we discuss the relevance of the above results to the laser wake field accelerator concept. The question that must be answered is whether or not self-guiding by this mechanism will occur at densities suitable for wakefield generation.

As mentioned in the introduction, the laser pulse width used in this concept determines the required density of free electrons. The converse is also true; higher density requires a shorter pulse width. This may be made more quantitative as follows. From the relationship between the laser pulsewidth and the plasma period, $\tau_{l}=\tau_{p}$, required for wake field acceleration, it can be seen that the resonant density is inversely proportional to the square of the pulse width, $\tau_{l}=2 \pi / \omega_{p} \propto n_{e}-1 / 2$. It should also be noted that the amplitude of the wake field increases linearly with the density, as given by Poison's equation: $\nabla \cdot E=4 \pi \rho=4 \pi\left(n_{i}-n_{e}\right) e$. Thus for the highest resonant density, and also wake field amplitude, the shortest laser pulse width should be used, assuming a fixed intensity. For the shortest pulsewidths currently attainable, $400 \mathrm{fs}$, still maintaining the intensity required for wakefield generation (see article on laser technology by Mourou and Umstadter in these Proceedings), the resonant density is $n_{e}=5 \times 10^{16} \mathrm{~cm}^{-3}$. We must now address issue of whether or not self-guiding at this density is possible.

The results shown above indicate that self-guiding is possible at densities of ... for a laser intensity of .... At higher intensities .... 


\section{SUMMARY}

In summary, the observations reported here, of self-focusing with multiple foci by a high-intensity subpicosecond laser, may provide a solution to the important problem of extending the focusing region in high-intensity laser wake field interactions. This multi-focusing mechanism may only be understood by considering both gas and plasma contributions to the index of refraction. However, a better understanding of the nonlinear properties of a gas in a high laser field is required. Finally, small-scale self-focusing due to beam non-uniformity remains the outstanding issue limiting self-guiding by this mechanism.

\section{ACKNOWLEDGEMENTS}

This work was partially funded by the National Science Foundation Center for Ultrafast Optical Science, contract \#PHY8920108. The authors thank C. Y. Chien for helpful assistance with the laser operation, and G. Mourou for his encouragement during the experiment.

\section{REFERENCES}

[1] G. Mourou and D. Umstadter, Development and Applications of Compact High-Intensity Lasers, Phys. Fluids B 4, 2315.

[2] T. Tajima and J. M. Dawson, Phys. Rev. Lett 43, 267 (1979); L. M. Gorbunov and V.I. Kirsanov, Zh. Eksp. Teor. Fiz. 93, 509 (1987) [Sov. Phys. JETP 66, 290 (1987)].

[3] A. B. Borisov, A. B. Borovskiy, B. B. Korobkin, A. M. Prokhorov, O. B. Shiryaev, X. M. Shi, T. S. Luk, A. McPherson, J. C. Solem, K. Boyer, and C. K. Rhodes, Phys. Rev. Lett. 68, 2309 (1992); A. B. Borisov, A. B. Borovskiy, O. B. Shiryaev, B. B. Korobkin, A. M. Prokhorov, J. C. Solem, T. S. Luk, K. Boyer, and C. K. Rhodes, Phys. Rev. A 45, 5830 (1992).

[4] P. Sprangle, E. Esarey and A. Ting, Phys. Rev. Lett., 23 Apr (1990); P. Sprangle, E. Esarey, A. Ting and G. Joyce, Appl. Phys. Lett. 53, 2146 (1988); A. Ting, E. Esarey, and P. Srangle, Phys. Fluids B 2, 1390 (1990); P. Sprangle, E. Esarey, and A. Ting, Phys. Rev. A 41, 4463 (1990); P. Sprangle, C. M. Tang and E. Esarey, IEEE Trans. Plasma Sci. PS-15, 145 (1987); E. Esarey, A. Ting, P. Sprangle, and G. Joyce, Comments on Plasma Phys. Controlled Fusion 12, 191 (1989).

[5] C. Max, "Physics of the Coronal Plasma in Laser Fusion Targets," in Laser-Plasma Interaction, R. Balian and J. C. Adam, eds. (North Holland, Amsterdam, 1982).

[6] C. Max, J. Arons and A. B. Langdon, Phys. Rev. Lett. 33, 209 (1974); G. Z. Sun, E. Ott, Y. C. Lee, and P. Guzdar, Phys. Fluids 30, 526 (1987); P. 
Sprangle, C. M. Tang, and E. Esarey, IEEE Trans. Plasma Sci. PS-15, 145 (1987). J. Solem, T. S. Luk, and K. Boyer, IEEE Jour. of Quant. Elect. 25, 2423 (1989).

[7] V. V. Korobkin and A. J. Alcock, Phys. Rev. Lett. 21, 1433 (1968). A. J. Alcock, C. DeMichelis, V. V. Korobkin, and M. C. Richardson, Appl. Phys. Lett. 14, 145 (1969). F. N. Bunkin, I. K. Krasyuk, V. M. Marchenko, P. P. Pashinin, and A. M. Prokhorov, Soviet Phys. JETP 33, 717 (1971).

[8] T. Afshar-rad, L. A. Gizzi, M. Desselberger, F. Khattak, O. Willi, and A. Giulietti, Phys. Rev. Lett. 68, 942 (1992); P. E. Young, H. A Baldis, T. W. Johnston, W. L. Kruer and K. G. Estabrook, Phys. Rev. Lett. 63, 2812 (1988); P. E. Young, R. P. Drake, E. M. Campbell and K. G. Estabrook, Phys Rev. Lett. 61, 2336 (1988). M. J. Herbst, J. A. Stamper, R. R. Whitlock, R. H. Lehmberg, and B. H. Ripin, Phys. Rev. Lett. 46, 328 (1981); R. Craxton and R. L. McCrory, J. Appl. Phys. 56, 108 (1984).

[9] Y. R. Shen, Principles of Nonlinear Optics (Wiley, New York, 1984).

[10] Y. Beaudoin, C. Y. Chien, S. Coe, J. Tapie, and G. Mourou, submitted to Optics Letters (1992).

[11] P. Maine, D. Strickland, P. Bado, M. Pessot and G. Mourou, IEEE J. Quantum Electron. QE-24, 398 (1988).

[12] O. Svelto, in Progress in Optics, E. Wolf, editor, North-Holland, Amsterdam, 1974, Vol. XII, pp. 1-51.

[13] I. Golub, Y. Beaudoin, and S. L. Chin, J. Opt. Soc. Am. B5, 2490 (1988)

[14] X. Liu, D. Umstadter, S. Coe, C. Chien, E. Esaray, and P. Sprangle, in OSA Proceedings on Short Wavelength Coherent Radiation: Generation and Applications, 1991, P. Bucksbaum and N. Ceglio, eds. (Optical Society of America, Washington, D.C., 1991), pp. 7-11.

[15] R. Dewhurst, J. Phys. D. Appl. Phys., 11, 191 (1978)

[16] G. M. Weyl, in Laser-Induced Plasmas and Applications, L. J. Radziemski and D. A. Cremers, editors, M. Dekker, New York, 1989, pp. 1-67.

[17] Y. Shimoji, A. Fay, R. Chang, and N. Djeu, J. Opt. Soc. Am. B6, 1994 (1989)

[18] W. P. Leemans, Topics in High Intensity Laser Plasma Interaction, $\mathrm{PhD}$. Thesis, (UCLA, Los Angeles, 1991) p. 59.

[19] J. Eberly, J. Javanainen, K. Rzazewski, Phys. Reports, 204 331-383 (1991)

[20] F. Brunel, J. Opt. Soc. Amer. B 7, 521 (1990). 


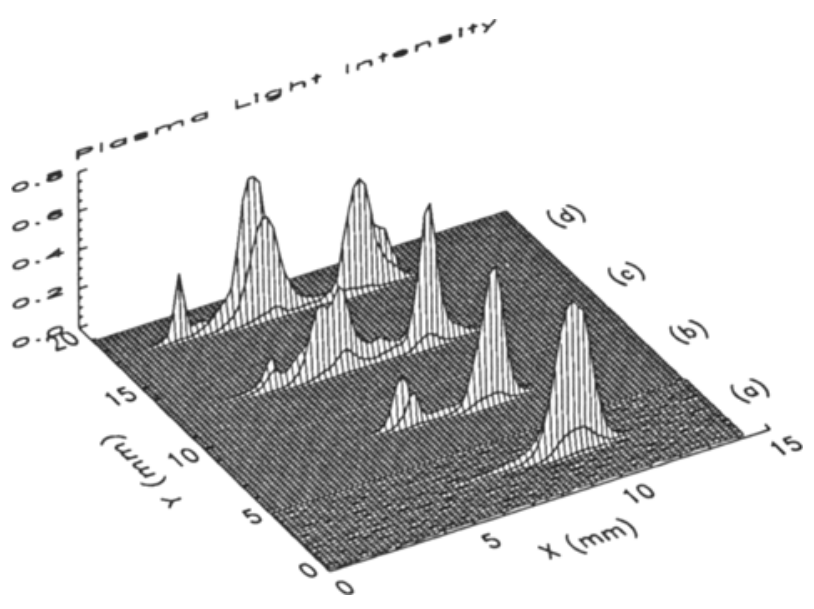

Figure 1: Normalized plasma column images with $f=20 \mathrm{~cm}$ lens in hydrogen showing multiple foci formation as pressure increases. The laser peak power for (b)-(d) was about $200 \mathrm{GW}$ ( $80 \mathrm{~mJ}$ per pulse). (a) $p=25$ Torr, Incident laser pulse energy $\sim 20 \mathrm{~mJ}$, focus position reference shot; (b) $p=50$ Torr; (c) $p=200$ Torr; (d) $p=350$ Torr. The beam propagates from left to right. 
D. Umstader and X. Liu 459

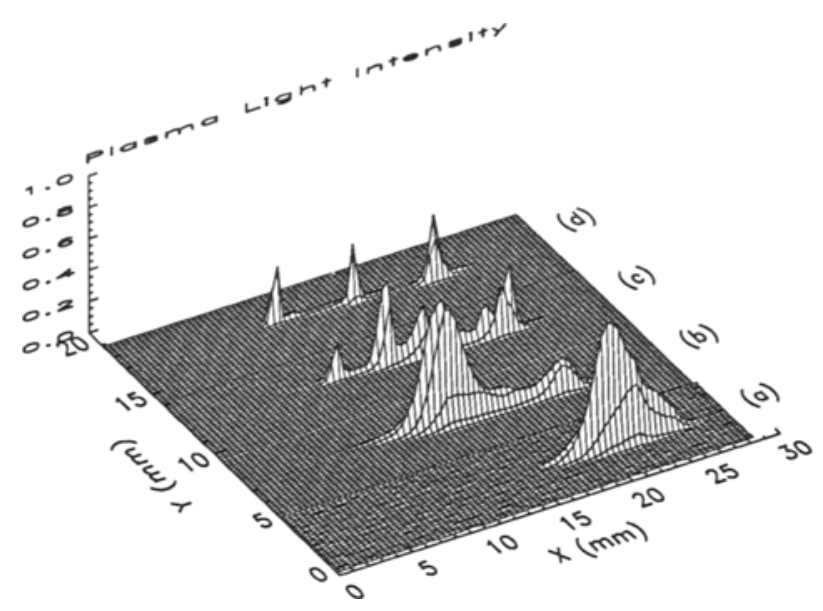

Figure 2: Normalized plasma column images with $f=40 \mathrm{~cm}$ lens in hydrogen. The laser peak power for (b)-(d) was again about $200 \mathrm{GW}$. (a) $p=30$ Torr, 40 $\mathrm{mJ}$; (b) $p=100$ Torr; (c) $p=250$ Torr; (d) $p=500$ Torr. The beam propagates from left to right.
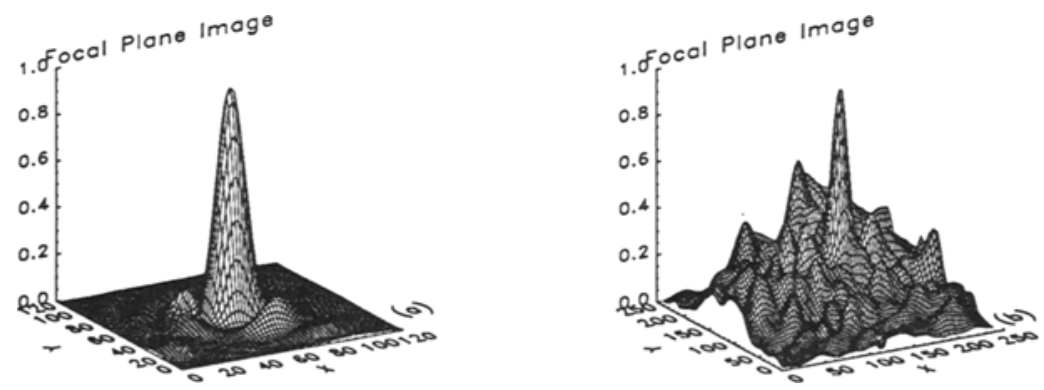

Figure 3: On-axis image of the laser focal plane showing beam break-up: (a) in vacuum, laser energy $10 \mathrm{~mJ}$; (b) $p=50$ Torr, $40 \mathrm{~mJ}$, the intensity in vacuum would be $7 \times 10^{15} \mathrm{~W} / \mathrm{cm}^{2}$. 


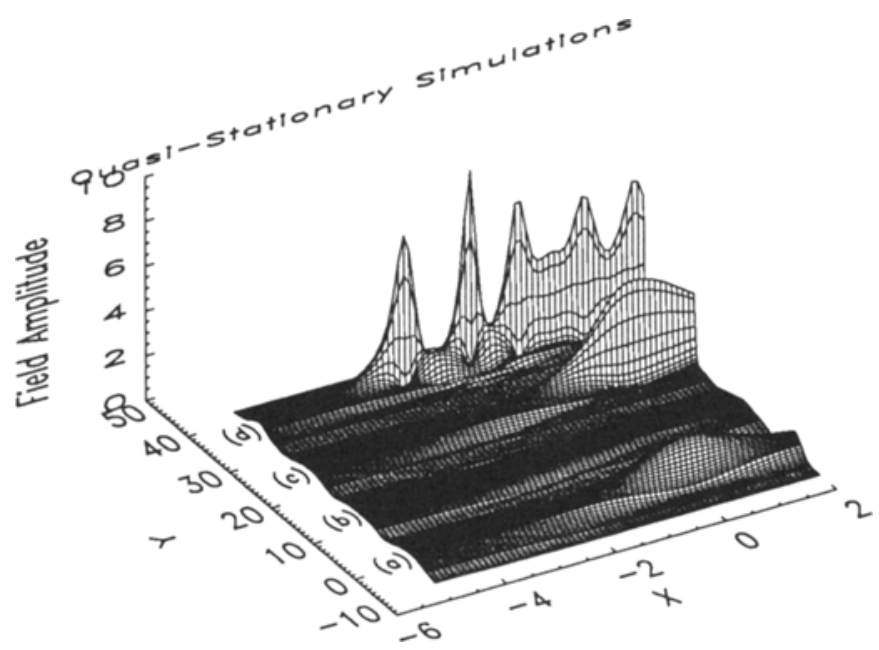

Figure 4: Numerical solution of Eq. (4). An $f / 10$ lens is used for $1 \mu \mathrm{m}$ laser wavelength. The $x$-axis is in units of vacuum Rayleigh range, the $y$-axis is in units of focused vacuum spot size. The laser field is in units of the ionization saturation field $E_{s}$, which is taken to be $2.9 \times 10^{6}$ esu (corresponding to saturation intensity $\left.I_{s}=10^{15} \mathrm{~W} / \mathrm{cm}^{2}\right)$. For all cases, the focused vacuum field strength is $E=6.5 \times 10^{6}$ esu $\left(I=5 \times 10^{15} \mathrm{~W} / \mathrm{cm}^{2}\right)$, and values of $n_{2}=n_{2}^{\prime} n_{t}$ are given for atmospheric pressure. For (b)-(d) the density is 100 Torr of hydrogen. (a) in vacuum, the beam comes to a focus at $x=0$; (b) $n_{2}=1.5 \times 10^{-16}$ esu (ref. [17]), the peak field strength is 3.5 times lower than in vacuum; (c) $n_{2}=7.1 \times 10^{-16}$ esu; (d) $n_{2}=1.0 \times 10^{-15}$ esu. 